\title{
The Optimal Control Problem with State Constraints for Fully Coupled Forward-Backward Stochastic Systems with Jumps
}

\author{
Qingmeng Wei \\ School of Mathematics and Statistics, Northeast Normal University, Changchun 130024, China \\ Correspondence should be addressed to Qingmeng Wei; qingmengwei@gmail.com
}

Received 26 November 2013; Accepted 22 December 2013; Published 13 February 2014

Academic Editor: Litan Yan

Copyright (C) 2014 Qingmeng Wei. This is an open access article distributed under the Creative Commons Attribution License, which permits unrestricted use, distribution, and reproduction in any medium, provided the original work is properly cited.

\begin{abstract}
We focus on the fully coupled forward-backward stochastic differential equations with jumps and investigate the associated stochastic optimal control problem (with the nonconvex control and the convex state constraint) along with stochastic maximum principle. To derive the necessary condition (i.e., stochastic maximum principle) for the optimal control, first we transform the fully coupled forward-backward stochastic control system into a fully coupled backward one; then, by using the terminal perturbation method, we obtain the stochastic maximum principle. Finally, we study a linear quadratic model.
\end{abstract}

\section{Introduction}

In finance, various stochastic models are applied to simulate the price movements of financial instruments. For example, in the Black-Scholes model for pricing options, the price process of financial instrument is described by a geometrical Brownian movement which is a continuous-time stochastic process. However, the sudden world events would cause larger fluctuation in asset prices, which is intuitive in financial markets. In this situation, the price process usually follows a stochastic equation with jumps; that is, the uncertainty of the model comes from not only a Brownian motion but also a Possion random measure. Accordingly, for this case, there are also many applications in finance which produce the associated optimal control problem, such as risk management and option prices. In this paper, we focus on the optimal control problem for the discontinuous case.

There are two important approaches in control theory, namely, stochastic maximum principle and dynamic programming principle, where the stochastic maximum principle presents the necessary condition for the solution of the controlled system. In stochastic control theory, the maximum principle for the optimal control has been developed rapidly, especially, along with the appearance of nonlinear backward stochastic differential equations (BSDEs, for short) introduced by Pardoux and Peng [1], by Kushner [2], Bismut [3], Bensoussan [4], Haussmann [5], Hu [6], Peng [7, 8], and Øksendal and Sulem [9] (see [10] for the complete bibliography). In 1993, Peng [8] researched the decoupled forward-backward stochastic control system with the control domain being convex and deduced the maximum principle. Since then, under different assumptions, a lot of works about this topic have been springing up (refer to [9, 11-20]).

For the models with jumps, Situ [21] first got the maximum principle for forward stochastic controlled system with the jump diffusion coefficient independent of the control variable. Tang and $\mathrm{Li}[22]$ obtained the maximum principle in global form, when both diffusion and jump coefficients depend on the control variable and some state constraints are imposed. For the control problem of decoupled forwardbackward stochastic differential equations (FBSDEs, for short) with jumps controlled system, the readers may refer to Øksendal and Sulem [9], Shi and Wu [23], and the references therein.

For the optimal control problem of the fully coupled FBSDEs with jumps, Shi and Wu [20] studied one kind of control problems with the control domain nonconvex, but the control variable does not appear in the diffusion and the jump coefficients of the forward equation. In [19], Shi discussed a general optimal control problem and 
derived Pontraygin's maximum principle when the control domain is nonconvex, and the control variable appears in both diffusion and jump coefficients of the forward equation.

This paper is concerned with the optimal control problem of the fully coupled FBSDE with jumps (9), where we restrict the forward terminal state $x(T)$ in a convex set $K$, the control variables are non-convex, and the controlled model is not continuous. Moreover, we allow the diffusion and jump coefficients $\bar{\sigma}, \bar{h}$ in the forward equation to depend on the two different control variables, which is mainly because two diffusion terms are needed when applying martingale representation theorem to determine the solution of the BSDE with jumps. The control variables $u(\cdot), v(\cdot, \cdot)$ associate with the diffusion term $\sigma$ and the jump diffusion $h$, respectively.

In order to deduce the stochastic maximum principle, we adopt the dual method (developed in [24]) and terminal perturbation method which are used to solve optimization problems with state constraints (refer to $[12-14,17,25-$ $28]$ ). Recently, the dual approach is applied to utility optimization problem with volatility and ambiguity (see [29, 30]). First we transform the FBSDE with jumps (9) into a pure backward controlled one with jumps, in which the terminal state $x(T)$ is regarded as the control variable which is much more easier to be dealt with than the initial state constraint. Meanwhile, the initial condition of the forward equation becomes an additional constraint. Fortunately, this constraint can be solved by applying Ekeland's variational principle.

The paper is structured as follows. In Section 2, for fully coupled FBSDEs with jumps, we present some preliminaries. In Subsection 2.1, we first formulate the optimal control problem. By proving the variational inequality, we derive a stochastic maximum principle which presents the necessary condition for the optimal terminal state. Section 3 studies an application in stochastic linear quadratic control problem.

\section{Preliminaries}

Let $\left(\Omega, \mathscr{F},\left\{\mathscr{F}_{t}\right\}_{t \geq 0}, P\right)$ be a complete probability space equipped with a natural filtration $\mathbb{F}=\left\{\mathscr{F}_{t}\right\}_{t \geq 0}$ which is generated by the following two mutually independent processes and completed by all $P$-null sets:

(i) a $d$-dimensional standard Brownian motion $\left\{B_{t}\right\}_{t \geq 0}$;

(ii) a Poisson random measure $\mu$ on $\mathbb{R}^{+} \times E$, where $E=$ $\mathbb{R}^{l} \backslash\{0\}$ is equipped with its Borel $\sigma$-field $\mathscr{B}(E)$, with the compensator $\widehat{\mu}(d t, d e)=d t \lambda(d e)$ such that $\{\widetilde{\mu}((0, t] \times A)=(\mu-\widehat{\mu})((0, t] \times A)\}_{t \geq 0}$ is a martingale for all $A \in \mathscr{B}(E)$ satisfying $\lambda(A)<\infty$. Here $\lambda$ is assumed to be a $\sigma$-finite Lévy measure on $(E, \mathscr{B}(E))$ with the property that $\int_{E}\left(1 \wedge|e|^{2}\right) \lambda(d e)<\infty$.
For any $n \geq 1,|z|$ denotes the Euclidean norm of $z \in \mathbb{R}^{n}$. $T>0$ is a fixed time horizon. Let us present the following spaces of processes which will be used later:

(i)

$$
\begin{aligned}
& \mathcal{S}^{2}\left(0, T ; \mathbb{R}^{n}\right) \\
& :=\left\{\psi \mid \psi: \Omega \times[0, T] \longrightarrow \mathbb{R}^{n} \text { is an } \mathbb{F}\right. \text {-adapted càdlàg process } \\
& \left.:\|\psi\|^{2}=E\left[\sup _{t \leq s \leq T}\left|\psi_{s}\right|^{2}\right]<+\infty\right\} ;
\end{aligned}
$$

(ii)

$$
\begin{aligned}
& \mathscr{M}^{2}\left(0, T ; \mathbb{R}^{n}\right) \\
& :=\left\{\varphi \mid \varphi: \Omega \times[0, T] \longrightarrow \mathbb{R}^{n} \text { is an } \mathbb{F}\right. \text {-predictable process } \\
& \left.:\|\varphi\|^{2}=E\left[\int_{t}^{T}\left|\varphi_{s}\right|^{2} d s\right]<+\infty\right\} ;
\end{aligned}
$$

(iii)

$$
\begin{aligned}
& \mathscr{K}_{\lambda}^{2}\left(0, T ; \mathbb{R}^{n}\right) \\
& :=\left\{K \mid K: \Omega \times[0, T] \times E \longrightarrow \mathbb{R}^{n} \text { is } \mathscr{P} \otimes \mathscr{B}(E)\right. \text {-measurable } \\
& \left.\quad:\|K\|^{2}=E\left[\int_{t}^{T} \int_{E}\left|K_{s}(e)\right|^{2} \lambda(d e) d s\right]<+\infty\right\} ;
\end{aligned}
$$

(iv)

$$
\begin{array}{r}
\mathscr{L}_{\lambda}^{2}\left(\mathbb{R}^{n}\right) \\
:=\left\{k \mid k: E \longrightarrow \mathbb{R}^{n} \text { is } \mathscr{B}(E)\right. \text {-measurable } \\
\left.:\|k\|^{2}=\int_{E}|k(e)|^{2} \lambda(d e)<+\infty\right\} ;
\end{array}
$$

(v)

$$
\begin{aligned}
L^{2}\left(\Omega, \mathscr{F}_{T}, P ; \mathbb{R}\right) & \\
& :=\left\{\xi: \Omega \longrightarrow \mathbb{R} \text { is } \mathscr{F}_{T} \text {-measurable }: E|\xi|^{2}<\infty\right\},
\end{aligned}
$$

where $\mathscr{P}$ denotes the $\sigma$-field of $\mathbb{F}$-predictable subsets of $\Omega \times$ $[0, T]$.

First we recall some results about fully coupled FBSDEs with jumps. Consider the following fully coupled FBSDEs with jumps:

$$
\begin{aligned}
& d x(t)=\bar{b}(t, x(t), y(t), z(t), k(t, \cdot)) d t \\
& +\bar{\sigma}(t, x(t), y(t), z(t), k(t, \cdot)) d W(t) \\
& +\int_{E} \bar{h}(t, x(t), y(t), z(t), k(t, e)) \tilde{\mu}(d t d e), \\
& \quad x(t)=\eta, \\
& d y(t)=-\bar{g}(t, x(t), y(t), z(t), k(t, \cdot)) d t \\
& \quad+z(t) d W(t)+\int_{E} k(t, e) \tilde{\mu}(d t d e), \\
& y(T)=\Phi(x(T)), \quad t \in[0, T],
\end{aligned}
$$


where $(x, y, z, k) \in \mathbb{R}^{n} \times \mathbb{R}^{m} \times \mathbb{R}^{m \times d} \times \mathscr{L}_{\lambda}^{2}\left(\mathbb{R}^{m}\right), T>0$,

$\bar{b}: \Omega \times[0, T] \times \mathbb{R}^{n} \times \mathbb{R}^{m} \times \mathbb{R}^{m \times d} \times \mathscr{L}_{\lambda}^{2}\left(\mathbb{R}^{m}\right) \rightarrow \mathbb{R}^{n}$, $\bar{\sigma}: \Omega \times[0, T] \times \mathbb{R}^{n} \times \mathbb{R}^{m} \times \mathbb{R}^{m \times d} \times \mathscr{L}_{\lambda}^{2}\left(\mathbb{R}^{m}\right) \rightarrow \mathbb{R}^{n \times d}$,

$\bar{h}: \Omega \times[0, T] \times \mathbb{R}^{n} \times \mathbb{R}^{m} \times \mathbb{R}^{m \times d} \times \mathbb{R}^{m} \rightarrow \mathbb{R}^{n \times d}, \bar{g}: \Omega \times$ $[0, T] \times \mathbb{R}^{n} \times \mathbb{R}^{m} \times \mathbb{R}^{m \times d} \times \mathscr{L}_{\lambda}^{2}\left(\mathbb{R}^{m}\right) \rightarrow \mathbb{R}^{m}$,

$\Phi: \Omega \times \mathbb{R}^{n} \rightarrow \mathbb{R}^{m}$

are $\mathbb{F}$-progressively measurable processes.

Here we adopt the monotonic condition for fully coupled FBSDEs with jumps developed by $\mathrm{Wu}$ [31].

Given an $m \times n$ full-rank matrix $G$, we define

$$
\lambda=\left(\begin{array}{l}
x \\
y \\
z
\end{array}\right), \quad A(t, \lambda, k(\cdot))=\left(\begin{array}{c}
-G^{T} \bar{g} \\
G \bar{b} \\
G \bar{\sigma}
\end{array}\right)(t, \lambda, k(\cdot))
$$

where $G^{T}$ is the transposed matrix of $G$.

We assume the following.

$\left(\mathrm{H}_{1}\right)(\mathrm{i})\langle A(t, \lambda, k(\cdot))-A(t, \bar{\lambda}, \bar{k}(\cdot)), \lambda-\bar{\lambda}\rangle+\int_{E}\langle G \widehat{h}(e)$, $\widehat{k}(e)\rangle \lambda(d e) \leq-\beta_{1}|G \hat{x}|^{2}-\beta_{2}\left(\left|G^{T} \hat{y}\right|^{2}+\left|G^{T} \widehat{z}\right|^{2}+\right.$ $\left.\int_{E}\left|G^{T} \widehat{k}(e)\right|^{2} \lambda(d e)\right)$,

(ii) $\langle\Phi(x)-\Phi(\bar{x}), G(x-\bar{x})\rangle \geq \mu_{1}|G \widehat{x}|^{2}, \widehat{x}=x-\bar{x}$, $\widehat{y}=y-\bar{y}, \widehat{z}=z-\bar{z}, \widehat{k}(\cdot)=k(\cdot)-\bar{k}(\cdot)$,

where $\beta_{1}, \beta_{2}, \mu_{1}$ are nonnegative constants with $\beta_{1}+$ $\beta_{2}>0, \beta_{2}+\mu_{1}>0$. Moreover, we have $\beta_{1}>0$, $\mu_{1}>0$ (resp., $\beta_{2}>0$ ), when $m>n$ (resp., $m<n$ ).

$\left(\mathrm{H}_{2}\right)$ For every $(x, y, z, k) \in \mathbb{R}^{n} \times \mathbb{R}^{m} \times \mathbb{R}^{m \times d} \times \mathbb{R}^{m}$, $\Phi(x) \in L^{2}\left(\Omega, \mathscr{F}_{T}, P ; \mathbb{R}^{m}\right), b, \sigma, h, f$ are progressively measurable and

$$
\begin{aligned}
E \int_{0}^{T}|b(s, 0,0,0,0)|^{2} d s+E \int_{0}^{T}|f(s, 0,0,0,0)|^{2} d s \\
\quad+E \int_{0}^{T}|\sigma(s, 0,0,0,0)|^{2} d s \\
\quad+E \int_{0}^{T} \int_{E}|h(s, 0,0,0,0, e)|^{2} \lambda(d e) d s<\infty
\end{aligned}
$$

Lemma 1. Under assumptions $\left(\mathrm{H}_{1}\right),\left(\mathrm{H}_{2}\right)$, for any $\eta \in$ $L^{2}\left(\Omega, \mathscr{F}_{t}, P ; R^{n}\right)$, FBSDE with jumps (6) has a unique adapted solution $(x(t), y(t), z(t), k(t, \cdot))_{t \in[0, T]} \in \mathscr{M}^{2}\left(0, T ; R^{n+m+m \times d}\right) \times$ $\mathscr{K}_{\lambda}^{2}\left(0, T ; \mathbb{R}^{m}\right)$.

For the proof, the readers may refer to $\mathrm{Wu}$ [31].

2.1. Problem Formulation. Denote $\mathscr{U}_{\mathrm{ad}}:=\left\{u(\cdot) \mid u(\cdot) \in \mathscr{M}^{2}\right.$ $\left.\left(0, T ; \mathbb{R}^{n \times d}\right)\right\}, \mathscr{V}_{\text {ad }}:=\left\{v(\cdot, \cdot) \mid v(\cdot, \cdot) \in \mathscr{K}_{\lambda}^{2}\left(0, T ; \mathbb{R}^{n}\right)\right\}$ by the set of admissible controls $u(\cdot), v(\cdot, \cdot)$, respectively.
For the given admissible control processes $u(\cdot) \in \mathscr{U}_{\text {ad }}$, $v(\cdot, \cdot) \in \mathscr{V}_{\text {ad }}$, the dynamic of control system is described by the following fully coupled FBSDEs with jumps:

$$
\begin{aligned}
d x(t)= & \bar{b}(t, x(t), y(t), z(t), k(t, \cdot), u(t), v(t, \cdot)) d t \\
& +\bar{\sigma}(t, x(t), y(t), z(t), k(t, \cdot), u(t)) d B_{t} \\
& +\int_{E} \bar{h}(t, x(t-), y(t-), z(t), \\
& k(t, e), v(t, e)) \tilde{\mu}(d t d e), \\
& x(0)=a, \\
d y(t)=-\bar{g}(t, x(t), y(t), z(t), k(t, \cdot), u(t), v(t, \cdot)) d t & +z(t) d B_{t}+\int_{E} k(t, e) \tilde{\mu}(d t d e), \\
& y(T)=\Phi(x(T)), \quad t \in[0, T],
\end{aligned}
$$

with $(x, y, z, k) \in \mathbb{R}^{n} \times \mathbb{R}^{m} \times \mathbb{R}^{m \times d} \times \mathbb{R}^{m}, T>0$, and

$\bar{b}:[0, T] \times \mathbb{R}^{n} \times \mathbb{R}^{m} \times \mathbb{R}^{m \times d} \times L_{\lambda}^{2}\left(\mathbb{R}^{m}\right) \times \mathbb{R}^{n \times d} \times L_{\lambda}^{2}\left(\mathbb{R}^{n}\right) \rightarrow$ $\mathbb{R}^{n}$

$\bar{\sigma}:[0, T] \times \mathbb{R}^{n} \times \mathbb{R}^{m} \times \mathbb{R}^{m \times d} \times L_{\lambda}^{2}\left(\mathbb{R}^{m}\right) \times \mathbb{R}^{n \times d} \rightarrow \mathbb{R}^{n \times d}$,

$\bar{h}:[0, T] \times \mathbb{R}^{n} \times \mathbb{R}^{m} \times \mathbb{R}^{m \times d} \times \mathbb{R}^{m} \times \mathbb{R}^{n} \rightarrow \mathbb{R}^{n}$,

$\bar{g}:[0, T] \times \mathbb{R}^{n} \times \mathbb{R}^{m} \times \mathbb{R}^{m \times d} \times L_{\lambda}^{2}\left(\mathbb{R}^{m}\right) \times \mathbb{R}^{n \times d} \times L_{\lambda}^{2}\left(\mathbb{R}^{n}\right) \rightarrow$ $\mathbb{R}^{m}, h: \mathbb{R}^{n} \rightarrow \mathbb{R}^{m}$.

$\left(\mathrm{H}_{3}\right)(\mathrm{i}) \bar{b}, \bar{\sigma}, \bar{h}, \bar{g}, \bar{l}, \psi$, and $\gamma$ are continuous in their arguments and continuously differentiable in $(x, y$, $z, k, u, v)$;

(ii) the derivatives of $\bar{b}, \bar{\sigma}, \bar{h}, \bar{g}$ in $(x, y, z, k, u, v)$ are bounded;

(iii) the derivatives of $\bar{l}$ in $(x, y, z, k, u, v)$ are bounded by $C(1+|x|+|y|+|z|+\|k\|+|u|+\|v\|)$, and the derivatives of $\psi$ and $\gamma$ in $x$ are bounded by $C(1+|x|)$.

Under $\left(\mathrm{H}_{1}\right)$ and $\left(\mathrm{H}_{3}\right)$, for any $u(\cdot) \in \mathcal{U}_{\mathrm{ad}}, v(\cdot, \cdot) \in \mathscr{V}_{\mathrm{ad}}$, from the existence and uniqueness theorem of fully coupled FBSDEs with jumps (refer to [31]), we know that (9) has a unique adapted solution $\Pi^{u, v}(\cdot):=\left(x^{u, v}(\cdot)\right.$, $\left.y^{u, v}(\cdot), z^{u, v}(\cdot), k^{u, v}(\cdot, \cdot)\right) \in \mathscr{M}^{2}\left(0, T ; \mathbb{R}^{n+m+m \times d}\right) \times \mathscr{K}_{\lambda}^{2}\left(0, T ; \mathbb{R}^{m}\right)$ so that the following cost functional $\bar{J}(u(\cdot), v(\cdot, \cdot))$ is well defined:

$$
\begin{gathered}
\bar{J}(u(\cdot), v(\cdot, \cdot)) \\
:=E\left[\int_{0}^{T} \bar{l}(t, x(t), y(t), z(t), k(t, \cdot),\right. \\
u(t), v(t, \cdot)) d t \\
+\psi(x(T))+\gamma(y(0))]
\end{gathered}
$$

where $\bar{l}:[0, T] \times \mathbb{R}^{n} \times \mathbb{R}^{m} \times \mathbb{R}^{m \times d} \times L_{\lambda}^{2}\left(\mathbb{R}^{m}\right) \times \mathbb{R}^{n \times d} \times L_{\lambda}^{2}\left(\mathbb{R}^{n}\right) \rightarrow$ $\mathbb{R}, \psi: \mathbb{R}^{n} \rightarrow \mathbb{R}, \gamma: \mathbb{R}^{m} \rightarrow \mathbb{R}$ 
Our optimal control problem with state constraints can be stated as follows.

Problem I. Minimize $\bar{J}(u(\cdot), v(\cdot, \cdot))$ subject to $u(\cdot) \in \mathscr{u}_{\mathrm{ad}}$, $v(\cdot, \cdot) \in \mathscr{V}_{\text {ad }}$, and $x(T) \in K$, where $K \subseteq \mathbb{R}^{n}$ is convex.

Remark 2. In our optimal control problem, the control variables are not convex. However, the terminal state is constrained in a convex set $K$. For the non-convex state constraint case, it is still an open problem.

2.2. Backward Formulation. In the above optimal control problem, the state constraint $x(T) \in K$ is new for fully coupled FBSDEs with jumps control system. To solve the state constraints, we adopt the dual method and terminal perturbation method. First we need to transform the discontinuous fully coupled forward-backward control system into a discontinuous backward form. In addition, the following assumption is necessary:

$\left(\mathrm{H}_{4}\right)$ there exists $\alpha_{1}, \alpha_{2}>0$, such that $\mid \sigma\left(t, x, y, z, k, u_{1}\right)-$ $\sigma\left(t, x, y, z, k, u_{2}\right)\left|\geq \alpha_{1}\right| u_{1}-u_{2} \mid$ and $\mid h\left(t, x, y, z, k, v_{1}\right)-$ $h\left(t, x, y, z, k, v_{2}\right)\left|\geq \alpha_{2}\right| v_{1}-v_{2} \mid$ for all $x \in \mathbb{R}^{n}, y \in \mathbb{R}^{m}$, $z \in \mathbb{R}^{m \times d}, k \in \mathbb{R}^{m}, t \in[0, T]$ and $u_{1}, u_{2} \in \mathbb{R}^{n \times d}$, $v_{1}, v_{2} \in \mathbb{R}^{n}$.

We point out that under $\left(\mathrm{H}_{3}\right)$ and $\left(\mathrm{H}_{4}\right)$, for any $(t, x) \epsilon$ $[0, T] \times \mathbb{R}^{n}, u$ and $\sigma(t, x, y, z, k, u), v$ and $h(t, x, y, z, k, v)$ are one-to-one correspondences. By letting $p \equiv \sigma(t, x, y, z, k, u)$, $q=h(t, x, y, z, k, v)$, we get the existence of the inverse functions $\widehat{\sigma}, \widehat{h}$ satisfying $u=\widehat{\sigma}(t, x, y, z, k, p), v=$ $\widehat{h}(t, x, y, z, k, q)$, respectively. So, (9) is rewritten as

$$
\begin{aligned}
d x(t)= & -b(t, x(t), y(t), z(t), k(t, \cdot), p(t), q(t, \cdot)) d t \\
+ & p(t) d B_{t}+\int_{E} q(t, e) \tilde{\mu}(d t d e), \\
& x(0)=a, \\
d y(t)=-g(t, x(t), y(t), z(t), k(t, \cdot), p(t), q(t, \cdot)) d t & \\
+ & z(t) d B_{t}+\int_{E} k(t, e) \tilde{\mu}(d t d e), \\
& y(T)=\Phi(x(T)), \quad t \in[0, T],
\end{aligned}
$$

where

$$
\begin{gathered}
b(t, x, y, z, k(t, \cdot), p, q(t, \cdot)) \\
=-\bar{b}(t, x, y, z, k(t, \cdot), \widehat{\sigma}(t, x, y, z, k(t, \cdot), p), \\
\widehat{h}(t, x, y, z, k(t, \cdot), q(t, \cdot))), \\
g(t, x, y, z, k(t, \cdot), p, q(t, \cdot)) \\
=\bar{g}(t, x, y, z, k(t, \cdot), \widehat{\sigma}(t, x, y, z, k(t, \cdot), p), \\
\widehat{h}(t, x, y, z, k(t, \cdot), q(t, \cdot))) .
\end{gathered}
$$

Then, combined with the existence and uniqueness theorem of fully coupled FBSDEs with jumps (refer to Lemma 1), we know it is equivalent to choose $p(\cdot), q(\cdot, \cdot)$ and select the terminal state $x(T)$. Thus, we obtain the following backward control system:

$$
\begin{aligned}
d x(t)= & -b(t, x(t), y(t), z(t), k(t, \cdot), p(t), q(t, \cdot)) d t \\
& +p(t) d B_{t}+\int_{E} q(t, e) \tilde{\mu}(d t d e), \\
& x(T)=\xi, \\
d y(t)=- & -g(t, x(t), y(t), z(t), k(t, \cdot), p(t), q(t, \cdot)) d t \\
& +z(t) d B_{t}+\int_{E} k(t, e) \tilde{\mu}(d t d e), \\
y(T)= & \Phi(\xi), \quad t \in[0, T],
\end{aligned}
$$

which associates with the following cost functional:

$$
\begin{gathered}
J(\xi):=E\left[\int_{0}^{T} l(t, x(t), y(t), z(t),\right. \\
k(t, \cdot), p(t), q(t, \cdot)) d t \\
+\psi(\xi)+\gamma(y(0))],
\end{gathered}
$$

where $\xi \in U$ is the control variable, $U=\left\{\left.\xi|E| \xi\right|^{2}<\infty, \xi \in\right.$ $K$, a.s. $\}$, and $l(t, x, y, z, k(t, \cdot), p, q(t, \cdot))=\bar{l}(t, x, y, z, k(t, \cdot)$, $\widehat{\sigma}(t, x, y, z, k(t, \cdot), p), \widehat{h}(t, x, y, z, k(t, \cdot), q(t, \cdot)))$.

Then, the optimal control problem is reformulated as follows.

Problem II. Minimize $J(u(\cdot), v(\cdot, \cdot))$ subject to $\xi \in U$ and $x^{\xi}(0)=a$.

We propose to consider Problem II, though the terminal state becomes a control variable and the initial condition $x^{\xi}(0)=a$ (which is the solution of (13) at time 0 under $\xi$ ) is regarded as a constraint. Even so, it is more relaxing to study Problem II than Problem I. Moreover, $b, g, l$ also satisfy the similar conditions in $\left(\mathrm{H}_{3}\right)$.

Henceforth, we convert to Problem II to prove the maximum principle for the optimal control $\xi^{*} \in U$.

2.3. Variational Equation. In this subsection, we will present the estimates for the variational equations to serve for the variational inequality.

First we define a metric in $U: d\left(\xi^{1}, \xi^{2}\right):=\left(E\left|\xi^{1}-\xi^{2}\right|^{2}\right)^{1 / 2}$, for $\xi^{1}, \xi^{2} \in U$. Obviously, $(U, d(\cdot, \cdot))$ is a complete metric space.

Suppose that $\xi^{*} \in U$ is an optimal control to Problem II and $\left(x^{*}(\cdot), p^{*}(\cdot), q^{*}(\cdot, \cdot), y^{*}(\cdot), z^{*}(\cdot), k^{*}(\cdot, \cdot)\right)$ is the associated state processes of (13) (with $\xi=\xi^{*}$ ). For each $0 \leq \rho \leq 1, \xi \in$ $U$, we know $\xi^{\rho}:=\xi^{*}+\rho\left(\xi-\xi^{*}\right) \in U$ (note $U$ is convex). And $\left(x_{\rho}(\cdot), p_{\rho}(\cdot), q_{\rho}(\cdot, \cdot), y_{\rho}(\cdot), z_{\rho}(\cdot), k_{\rho}(\cdot, \cdot)\right)$ denotes the state processes of (13) corresponding to $\xi=\xi^{\rho}$. 
Consider the following BSDEs with jumps:

$$
\begin{gathered}
d \widehat{x}(t)=-\left[b_{x}^{*}(t, \cdot) \hat{x}(t)+b_{y}^{*}(t, \cdot) \hat{y}(t)+b_{z}^{*}(t, \cdot) \hat{z}(t)\right. \\
+b_{k}^{*}(t, \cdot) \hat{k}(t, \cdot)+b_{p}^{*}(t, \cdot) \widehat{p}(t) \\
\left.+b_{q}^{*}(t, \cdot) \widehat{q}(t, \cdot)\right] d t \\
+\widehat{p}(t) d B_{t}+\int_{E} \widehat{q}(t, e) \widetilde{\mu}(d t d e), \\
\widehat{x}(T)=\xi-\xi^{*}, \\
d \widehat{y}(t)=-\left[g_{x}^{*}(t, \cdot) \widehat{x}(t)+g_{y}^{*}(t, \cdot) \hat{y}(t)\right. \\
+g_{z}^{*}(t, \cdot) \widehat{z}(t)+g_{k}^{*}(t, \cdot) \widehat{k}(t, \cdot) \\
\left.+g_{p}^{*}(t, \cdot) \hat{p}(t)+g_{q}^{*}(t, \cdot) \widehat{q}(t, \cdot)\right] d t \\
+\widehat{z}(t) d B_{t}+\int_{E} \widehat{k}(t, e) \widetilde{\mu}(d t d e), \\
\widehat{y}(T)=\Phi_{x}\left(\xi^{*}\right)\left(\xi-\xi^{*}\right), \quad t \in[0, T],
\end{gathered}
$$

where $b_{a}^{*}(t, \cdot)=b_{a}\left(t, x^{*}(t), y^{*}(t), z^{*}(t), k^{*}(t, \cdot), p^{*}(t)\right.$, $\left.q^{*}(t, \cdot)\right), g_{a}^{*}(t, \cdot)=g_{a}\left(t, x^{*}(t), y^{*}(t), z^{*}(t), k^{*}(t, \cdot), p^{*}(t), q^{*}(t\right.$, $\cdot)), a=x, p, q, y, z, k$, respectively. Equation (15) is regarded as the variational equation along with the optimal control $\xi^{*}$ and the state $\left(x^{*}(\cdot), p^{*}(\cdot), q^{*}(\cdot, \cdot), y^{*}(\cdot), z^{*}(\cdot), k^{*}(\cdot, \cdot)\right)$.

We point that (15) is composed of two fully coupled linear BSDEs with jumps. In fact, under $\left(\mathrm{H}_{3}\right)$ and $\left(\mathrm{H}_{4}\right)$, from the existence and uniqueness theorem for BSDE with jumps (Lemma 1 in [32]), we know that (15) has the unique adapted solution $(\widehat{x}(\cdot), \widehat{p}(\cdot), \widehat{q}(\cdot, \cdot)) \in \mathscr{M}^{2}\left(0, T ; \mathbb{R}^{n} \times \mathbb{R}^{n \times d}\right) \times$ $\mathscr{K}_{\lambda}^{2}\left(0, T ; \mathbb{R}^{n}\right),(\widehat{y}(\cdot), \widehat{z}(\cdot), \widehat{k}(\cdot, \cdot)) \in \mathscr{M}^{2}\left(0, T ; \mathbb{R}^{m} \times \mathbb{R}^{m \times d}\right) \times$ $\mathscr{K}_{\lambda}^{2}\left(0, T ; \mathbb{R}^{m}\right)$.

For convenience, we denote

$$
\begin{gathered}
\tilde{x}_{\rho}(t)=\rho^{-1}\left[x_{\rho}(t)-x^{*}(t)\right]-\widehat{x}(t), \\
\tilde{p}_{\rho}(t)=\rho^{-1}\left[p_{\rho}(t)-p^{*}(t)\right]-\widehat{p}(t), \\
\tilde{q}_{\rho}(t, e)=\rho^{-1}\left[q_{\rho}(t, e)-q^{*}(t, e)\right]-\widehat{q}(t, e), \\
\tilde{y}_{\rho}(t)=\rho^{-1}\left[y_{\rho}(t)-y^{*}(t)\right]-\widehat{y}(t), \\
\widetilde{z}_{\rho}(t)=\rho^{-1}\left[z_{\rho}(t)-z^{*}(t)\right]-\widehat{z}(t), \\
\widetilde{k}_{\rho}(t, e)=\rho^{-1}\left[k_{\rho}(t, e)-k^{*}(t, e)\right]-\widehat{k}(t, e) .
\end{gathered}
$$

Then, we get the following results.
Lemma 3. Under $\left(\mathrm{H}_{1}\right),\left(\mathrm{H}_{3}\right)$, and $\left(\mathrm{H}_{4}\right)$, one has

$$
\begin{gathered}
\lim _{\rho \rightarrow 0} \sup _{0 \leq t \leq T} E\left|\tilde{x}_{\rho}(t)\right|^{2}=0, \\
\lim _{\rho \rightarrow 0} E\left[\int_{0}^{T}\left|\tilde{p}_{\rho}(t)\right|^{2} d t\right]=0, \\
\lim _{\rho \rightarrow 0} E\left[\int_{0}^{T} \int_{E}\left|\widetilde{q}_{\rho}(t, e)\right|^{2} \lambda(d e) d t\right]=0, \\
\lim _{\rho \rightarrow 0} \sup _{0 \leq t \leq T} E\left|\tilde{y}_{\rho}(t)\right|^{2}=0, \\
\lim _{\rho \rightarrow 0} E\left[\int_{0}^{T}\left|\tilde{z}_{\rho}(t)\right|^{2} d t\right]=0, \\
\lim _{\rho \rightarrow 0} E\left[\int_{0}^{T} \int_{E}\left|\tilde{k}_{\rho}(t, e)\right|^{2} \lambda(d e) d t\right]=0 .
\end{gathered}
$$

Proof. From (13), (15), and the notations (16), we have

$$
\begin{aligned}
& d \widetilde{x}_{\rho}(t)=-\rho^{-1}\left[b_{\rho}(t, \cdot)-b^{*}(t, \cdot)\right. \\
& -\rho b_{x}^{*}(t, \cdot) \hat{x}(t)-\rho b_{y}^{*}(t, \cdot) \hat{y}(t) \\
& -\rho b_{z}^{*}(t, \cdot) \widehat{z}(t)-\rho b_{k}^{*}(t, \cdot) \widehat{k}(t, \cdot) \\
& \left.-\rho b_{p}^{*}(t, \cdot) \hat{p}(t)-\rho b_{q}^{*}(t, \cdot) \hat{q}(t, \cdot)\right] d t \\
& +\tilde{p}_{\rho}(t) d B_{t}+\int_{E} \widetilde{q}_{\rho}(t, e) \widetilde{\mu}(d t d e) \\
& =-\left[b_{x}^{\rho}(t, \cdot) \tilde{x}_{\rho}(t)+b_{y}^{\rho}(t, \cdot) \tilde{y}_{\rho}(t)\right. \\
& +b_{z}^{\rho}(t, \cdot) \widetilde{z}_{\rho}(t)+b_{k}^{\rho}(t, \cdot) \widetilde{k}_{\rho}(t, \cdot) \\
& +b_{p}^{\rho}(t, \cdot) \tilde{p}_{\rho}(t)+b_{q}^{\rho}(t, \cdot) \tilde{q}_{\rho}(t, \cdot) \\
& \left.+A^{\rho}(t, \cdot)\right] d t+\tilde{p}_{\rho}(t) d B_{t} \\
& +\int_{E} \tilde{q}_{\rho}(t, e) \tilde{\mu}(d t d e), \\
& \tilde{x}_{\rho}(T)=0, \\
& d \tilde{y}_{\rho}(t)=-\rho^{-1}\left[g_{\rho}(t, \cdot)-g^{*}(t, \cdot)-\rho g_{x}^{*}(t, \cdot) \hat{x}(t)\right. \\
& -\rho g_{y}^{*}(t, \cdot) \hat{y}(t)-\rho g_{z}^{*}(t, \cdot) \widehat{z}(t) \\
& -\rho g_{k}^{*}(t, \cdot) \hat{k}(t, \cdot)-\rho g_{p}^{*}(t, \cdot) \widehat{p}(t) \\
& \left.-\rho g_{q}^{*}(t, \cdot) \widehat{q}(t, \cdot)\right] d t+\widetilde{z}_{\rho}(t) d B_{t}
\end{aligned}
$$




$$
\begin{gathered}
+\int_{E} \tilde{k}_{\rho}(t, e) \tilde{\mu}(d t d e) \\
=-\left[g_{x}^{\rho}(t, \cdot) \tilde{x}_{\rho}(t)+g_{y}^{\rho}(t, \cdot) \tilde{y}_{\rho}(t)\right. \\
\quad+g_{z}^{\rho}(t, \cdot) \tilde{z}_{\rho}(t)+g_{k}^{\rho}(t, \cdot) \tilde{k}_{\rho}(t, \cdot) \\
\quad+g_{p}^{\rho}(t, \cdot) \tilde{p}_{\rho}(t)+g_{q}^{\rho}(t, \cdot) \tilde{q}_{\rho}(t, \cdot) \\
\left.\quad+B^{\rho}(t, \cdot)\right] d t+\widetilde{z}_{\rho}(t) d B_{t} \\
+\int_{E} \tilde{k}_{\rho}(t, e) \tilde{\mu}(d t d e), \\
\tilde{y}_{\rho}(T)=\rho^{-1}\left[\Phi\left(\xi^{\rho}\right)-\Phi\left(\xi^{*}\right)\right]-\Phi_{x}\left(\xi^{*}\right)\left(\xi-\xi^{*}\right),
\end{gathered}
$$

where

$$
\begin{aligned}
& b_{a}^{\rho}(t, \cdot)=\int_{0}^{1} b_{a}\left(t, A_{x}(\alpha, t, \rho), A_{y}(\alpha, t, \rho),\right. \\
& A_{z}(\alpha, t, \rho), A_{k}(\alpha, t, \rho, \cdot), \\
& \left.A_{p}(\alpha, t, \rho), A_{q}(\alpha, t, \rho, \cdot)\right) d \alpha, \\
& g_{a}^{\rho}(t, \cdot)=\int_{0}^{1} g_{a}\left(t, A_{x}(\alpha, t, \rho), A_{y}(\alpha, t, \rho),\right. \\
& A_{z}(\alpha, t, \rho), A_{k}(\alpha, t, \rho, \cdot) \\
& \left.A_{p}(\alpha, t, \rho), A_{q}(\alpha, t, \rho, \cdot)\right) d \alpha, \\
& A^{\rho}(t, \cdot)=\left[b_{x}^{\rho}(t, \cdot)-b_{x}^{*}(t, \cdot)\right] \widehat{x}(t) \\
& +\left[b_{y}^{\rho}(t, \cdot)-b_{y}^{*}(t, \cdot)\right] \widehat{y}(t) \\
& +\left[b_{z}^{\rho}(t, \cdot)-b_{z}^{*}(t, \cdot)\right] \widehat{z}(t) \\
& +\left[b_{k}^{\rho}(t, \cdot)-b_{k}^{*}(t, \cdot)\right] \widehat{k}(t, \cdot) \\
& +\left[b_{p}^{\rho}(t, \cdot)-b_{p}^{*}(t, \cdot)\right] \widehat{p}(t) \\
& +\left[b_{q}^{\rho}(t, \cdot)-b_{q}^{*}(t, \cdot)\right] \widehat{q}(t, \cdot), \\
& B^{\rho}(t, \cdot)=\left[g_{x}^{\rho}(t, \cdot)-g_{x}^{*}(t, \cdot)\right] \widehat{x}(t) \\
& +\left[g_{y}^{\rho}(t, \cdot)-g_{y}^{*}(t, \cdot)\right] \widehat{y}(t) \\
& +\left[g_{z}^{\rho}(t, \cdot)-g_{z}^{*}(t, \cdot)\right] \widehat{z}(t) \\
& +\left[g_{k}^{\rho}(t, \cdot)-g_{k}^{*}(t, \cdot)\right] \widehat{k}(t, \cdot) \\
& +\left[g_{p}^{\rho}(t, \cdot)-g_{p}^{*}(t, \cdot)\right] \widehat{p}(t) \\
& +\left[g_{q}^{\rho}(t, \cdot)-g_{q}^{*}(t, \cdot)\right] \widehat{q}(t, \cdot), a \\
& =x, p, q, y, z, k \text {, resp. }
\end{aligned}
$$

$$
\begin{gathered}
A_{x}(\alpha, t, \rho)=x^{*}(t)+\alpha \rho\left(\widehat{x}(t)+\tilde{x}_{\rho}(t)\right), \\
A_{p}(\alpha, t, \rho)=p^{*}(t)+\alpha \rho\left(\widehat{p}(t)+\widetilde{p}_{\rho}(t)\right), \\
A_{q}(\alpha, t, \rho, \cdot)=q^{*}(t, \cdot)+\alpha \rho\left(\widehat{q}(t, \cdot)+\widetilde{q}_{\rho}(t, \cdot)\right), \\
A_{y}(\alpha, t, \rho)=y^{*}(t)+\alpha \rho\left(\widehat{y}(t)+\tilde{y}_{\rho}(t)\right), \\
A_{z}(\alpha, t, \rho)=z^{*}(t)+\alpha \rho\left(\widehat{z}(t)+\widetilde{z}_{\rho}(t)\right), \\
A_{k}(\alpha, t, \rho, \cdot)=k^{*}(t, \cdot)+\alpha \rho\left(\widehat{k}(t, \cdot)+\tilde{k}_{\rho}(t, \cdot)\right) .
\end{gathered}
$$

Applying Itô's formula to $\left|\widetilde{x}_{\rho}(t)\right|^{2}+\left|\widetilde{y}_{\rho}(t)\right|^{2}$, from $\left(\mathrm{H}_{3}\right),\left(\mathrm{H}_{4}\right)$, we obtain

$$
\begin{aligned}
& E\left|\tilde{x}_{\rho}(t)\right|^{2}+E\left|\tilde{y}_{\rho}(t)\right|^{2}+E \int_{t}^{T}\left|\tilde{p}_{\rho}(s)\right|^{2} d s \\
& +E \int_{t}^{T} \int_{E}\left|\tilde{q}_{\rho}(s, e)\right|^{2} \lambda(d e) d s \\
& +E \int_{t}^{T}\left|\tilde{z}_{\rho}(s)\right|^{2} d s \\
& +E \int_{t}^{T} \int_{E}\left|\widetilde{k}_{\rho}(s, e)\right|^{2} \lambda(d e) d s \\
& =E\left|\tilde{y}_{\rho}(T)\right|^{2} \\
& +2 E \int_{t}^{T} \tilde{x}_{\rho}(s)\left[b_{x}^{\rho}(s, \cdot) \tilde{x}_{\rho}(s)+b_{y}^{\rho}(t, \cdot) \tilde{y}_{\rho}(t)\right. \\
& +b_{z}^{\rho}(t, \cdot) \widetilde{z}_{\rho}(t)+b_{k}^{\rho}(t, \cdot) \tilde{k}_{\rho}(t, \cdot) \\
& +b_{p}^{\rho}(s, \cdot) \tilde{p}_{\rho}(s)+b_{q}^{\rho}(s, \cdot) \tilde{q}_{\rho}(s, \cdot) \\
& \left.+A^{p}(s, \cdot)\right] d s \\
& +2 E \int_{t}^{T} \widetilde{y}_{\rho}(s)\left[g_{x}^{\rho}(s, \cdot) \tilde{x}_{\rho}(s)\right. \\
& +g_{y}^{\rho}(t, \cdot) \tilde{y}_{\rho}(t)+g_{z}^{\rho}(t, \cdot) \tilde{z}_{\rho}(t) \\
& +g_{k}^{\rho}(t, \cdot) \widetilde{k}_{\rho}(t, \cdot)+g_{p}^{\rho}(s, \cdot) \tilde{p}_{\rho}(s) \\
& \left.+g_{q}^{\rho}(s, \cdot) \widetilde{q}_{\rho}(s, \cdot)+B^{p}(s, \cdot)\right] d s \\
& \leq E\left|\tilde{y}_{\rho}(T)\right|^{2} \\
& +C E \int_{t}^{T}\left(\left|\tilde{x}_{p}(s)\right|^{2}+\left|\tilde{y}_{p}(s)\right|^{2}\right) d s \\
& +C_{1} E \int_{t}^{T}\left(\left|\tilde{z}_{\rho}(s)\right|^{2}+\left|\tilde{p}_{\rho}(s)\right|^{2}\right) d s
\end{aligned}
$$




$$
\begin{aligned}
& +C_{1} E \int_{t}^{T} \int_{E}\left(\left|\widetilde{k}_{\rho}(s, e)\right|^{2}+\left|\widetilde{q}_{\rho}(s, e)\right|^{2}\right) \lambda(d e) d s \\
& +C_{2} \int_{t}^{T}\left(\left|A^{\rho}(s, \cdot)\right|^{2}+\left|B^{\rho}(s, \cdot)\right|^{2}\right) d s,
\end{aligned}
$$

with $C_{1}<1$. Furthermore, from Gronwall's inequality, we have

$$
\begin{aligned}
\sup _{0 \leq t \leq T} E & \left(\left|\widetilde{x}_{\rho}(t)\right|^{2}+\left|\tilde{y}_{\rho}(t)\right|^{2}\right) \\
& +E \int_{0}^{T}\left(\left|\widetilde{z}_{\rho}(s)\right|^{2}+\left|\widetilde{p}_{\rho}(s)\right|^{2}\right) d s \\
& +E \int_{t}^{T} \int_{E}\left(\left|\widetilde{k}_{\rho}(s, e)\right|^{2}+\left|\widetilde{q}_{\rho}(s, e)\right|^{2}\right) d s \\
\leq & \widehat{C} E\left|\tilde{y}_{\rho}(T)\right|^{2} \\
& +\widehat{C} E \int_{0}^{T}\left(\left|A^{\rho}(s, \cdot)\right|^{2}+\left|B^{\rho}(s, \cdot)\right|^{2}\right) d s .
\end{aligned}
$$

From Lebesgue's dominated convergence theorem, we know $\lim _{\rho \rightarrow 0} E \int_{0}^{T}\left(\left|A^{\rho}(s, \cdot)\right|^{2}+\left|B^{\rho}(s, \cdot)\right|^{2}\right) d t=0$. Also, it is clear that $\lim _{\rho \rightarrow 0} E\left|\tilde{y}_{\rho}(T)\right|^{2}=\lim _{\rho \rightarrow 0}\left(\rho^{-1}\left[\Phi\left(\xi^{\rho}\right)-\Phi\left(\xi^{*}\right)\right]-\Phi_{x}\left(\xi^{*}\right)(\xi-\right.$ $\left.\left.\xi^{*}\right)\right)=0$.

So, letting $\rho \rightarrow 0$ in (21), we complete the proof.

2.4. Variational Inequality. In this section we are going to explore the variational inequality for the variational equation (15). Let us start with the following Ekeland's variational principle [33] used to deal with the initial constraint $x^{\xi}(0)=$ a.

Lemma 4 (Ekeland's variational principle). Suppose that $(V, d(\cdot, \cdot))$ is a complete metric space and $F(\cdot): V \rightarrow \mathbb{R}$ is a proper lower semicontinuous function bounded from below. If, for some $\varepsilon>0$, there exists $u \in V$ satisfying $F(u) \leq$ $\inf _{v \in V} F(v)+\varepsilon$, then there exists $u_{\varepsilon} \in V$ such that

(i) $F\left(u_{\varepsilon}\right) \leq F(u)$

(ii) $d\left(u, u_{\varepsilon}\right) \leq \varepsilon$,

(iii) $F(v)+\sqrt{\varepsilon} d\left(v, u_{\varepsilon}\right) \geq F\left(u_{\varepsilon}\right), \forall v \in V$.

Given the optimal control $\xi^{*} \in U$, for any $\epsilon>0$, we introduce the penalty function $F_{\epsilon}(\cdot): U \rightarrow \mathbb{R}$ as

$$
\begin{aligned}
F_{\epsilon}(\xi)= & \left\{\left|x^{\xi}(0)-a\right|^{2}+\left(\max \left(0, \psi(\xi)-\psi\left(\xi^{*}\right)+\epsilon\right)\right)^{2}\right. \\
& +\left(\max \left(0, \gamma\left(y^{\xi}(0)\right)-\gamma\left(y^{*}(0)\right)+\epsilon\right)\right)^{2} \\
& \left.+\left(\max \left(0, \int_{0}^{T} l^{\xi}(t, \cdot) d t-\int_{0}^{T} l^{*}(t, \cdot) d t+\epsilon\right)\right)^{2}\right\}^{1 / 2},
\end{aligned}
$$

where $l^{\xi}(t, \cdot):=l\left(t, x^{\xi}(t), y^{\xi}(t), z^{\xi}(t), k^{\xi}(t, \cdot), p^{\xi}(t), q^{\xi}(t, \cdot)\right)$, $l^{*}(t, \cdot):=l\left(t, x^{*}(t), y^{*}(t), z^{*}(t), k^{*}(t, \cdot), p^{*}(t), q^{*}(t, \cdot)\right)$.

Remark 5. Note that under $\left(\mathrm{H}_{3}\right)$ the mappings $\left|x^{\xi}(0)-a\right|^{2}$, $\gamma\left(y^{\xi}(0)\right), \quad \psi(\xi), \quad l\left(t, x^{\xi}(t), y^{\xi}(t), z^{\xi}(t), k^{\xi}(t, \cdot), p^{\xi}(t), q^{\xi}(t, \cdot)\right)$ defined on $U$ are continuous mainly due to the continuous dependence of solutions of BSDE with jumps.

Theorem 6. Suppose $\left(\mathrm{H}_{3}\right),\left(\mathrm{H}_{4}\right)$ hold. If $\xi^{*}$ is optimal to Problem II, then there exist $h_{0} \in \mathbb{R}^{n}$ and $h_{1}, h_{2}, h_{3} \in \mathbb{R}$, with $h_{1}, h_{2}, h_{3} \geq 0$ and $\left|h_{0}\right|+\left|h_{1}\right|+\left|h_{2}\right|+\left|h_{3}\right| \neq 0$, such that the following variational inequality holds:

$$
\begin{aligned}
\left\langle h_{0}, \widehat{x}(0)\right\rangle+h_{1}\left\langle\psi_{x}\left(\xi^{*}\right), \xi-\xi^{*}\right\rangle+h_{2}\left\langle\gamma_{y}\left(y^{*}(0)\right), \widehat{y}(0)\right\rangle \\
\quad+h_{3} \int_{0}^{T}\left\langle l_{x}^{*}(t, \cdot), \widehat{x}(t)\right\rangle d t+h_{3} \int_{0}^{T}\left\langle l_{p}^{*}(t, \cdot), \widehat{p}(t)\right\rangle d t \\
+h_{3} \int_{0}^{T} \int_{E}\left\langle l_{q}^{*}(t, e), \widehat{q}(t, e)\right\rangle \lambda(d e) d t \\
+h_{3} \int_{0}^{T}\left\langle l_{y}^{*}(t, \cdot), \widehat{y}(t)\right\rangle d t+h_{3} \int_{0}^{T}\left\langle l_{z}^{*}(t, \cdot), \widehat{z}(t)\right\rangle d t \\
+h_{3} \int_{0}^{T} \int_{E}\left\langle l_{k}^{*}(t, e), \widehat{k}(t, e)\right\rangle \lambda(d e) d t \geq 0,
\end{aligned}
$$$$
\text { for any } \xi \in U \text {, }
$$

where $l_{a}^{*}(t, \cdot)=l_{a}\left(t, x^{*}(t), y^{*}(t), z^{*}(t), k^{*}(t, \cdot), p^{*}(t), q^{*}(t, \cdot)\right)$, $a=x, p, q, y, z, k$, respectively, and $(\widehat{x}(\cdot), \widehat{p}(\cdot), \widehat{q}(\cdot, \cdot)$, $\widehat{y}(\cdot), \widehat{z}(\cdot), \widehat{k}(\cdot, \cdot))$ is the solution of $(15)$.

Proof. Clearly, the following properties hold for the penalty function $F_{\epsilon}$ :

$$
\begin{gathered}
F_{\epsilon}\left(\xi^{*}\right)=\sqrt{3} \epsilon ; \quad F_{\epsilon}(\xi)>0, \quad \forall \xi \in U ; \\
F_{\epsilon}\left(\xi^{*}\right) \leq \inf _{\xi \in U} F_{\epsilon}(\xi)+\sqrt{3} \epsilon .
\end{gathered}
$$

Combined with Ekeland's variational principle (refer to Lemma 4), we obtain the existence of $\xi^{\epsilon} \in U$ satisfying

(i) $F_{\epsilon}\left(\xi^{\epsilon}\right) \leq F_{\epsilon}\left(\xi^{*}\right)$;

(ii) $d\left(\xi^{*}, \xi^{\epsilon}\right) \leq \sqrt{3} \epsilon$

(iii) $F_{\epsilon}(\xi)+\sqrt{\sqrt{3}} \epsilon d\left(\xi, \xi^{\epsilon}\right) \geq F_{\epsilon}\left(\xi^{\epsilon}\right), \forall \xi \in U$.

For $\forall \xi \in U, 0 \leq \rho \leq 1$, we have $\xi_{\rho}^{\epsilon}=\xi^{\epsilon}+\rho\left(\xi-\xi^{\epsilon}\right) \in U$. The solution of (13) with $\xi=\xi_{\rho}^{\epsilon}$ (resp., $\xi^{\epsilon}$ ) is denoted by $\left(x_{\rho}^{\epsilon}(\cdot), p_{\rho}^{\epsilon}(\cdot), q_{\rho}^{\epsilon}(\cdot, \cdot), y_{\rho}^{\epsilon}(\cdot), z_{\rho}^{\epsilon}(\cdot), k_{\rho}^{\epsilon}(\cdot, \cdot)\right) \quad\left(\right.$ resp., $x^{\epsilon}(\cdot), p^{\epsilon}(\cdot), q^{\epsilon}$ $\left.(\cdot, \cdot), y^{\epsilon}(\cdot), z^{\epsilon}(\cdot), k^{\epsilon}(\cdot, \cdot)\right)$. And $\left(\widehat{x}^{\epsilon}(\cdot), \widehat{p}^{\epsilon}(\cdot), \widehat{q}^{\epsilon}(\cdot, \cdot), \widehat{y}^{\epsilon}(\cdot), \widehat{z}^{\epsilon}(\cdot)\right.$, $\left.\widehat{k}^{\epsilon}(\cdot, \cdot)\right)$ denotes the solution of (15) with $\xi^{*}=\xi^{\epsilon}$. So,

$$
F_{\epsilon}\left(\xi_{\rho}^{\epsilon}\right)-F_{\epsilon}\left(\xi^{\epsilon}\right)+\sqrt{\sqrt{3}} \epsilon d\left(\xi_{\rho}^{\epsilon}, \xi^{\epsilon}\right) \geq 0 .
$$


Similar to the proof of Lemma 3, we have

$$
\begin{gathered}
\lim _{\rho \rightarrow 0} \sup _{0 \leq t \leq T} E\left|\rho^{-1}\left(x_{\rho}^{\epsilon}(t)-x^{\epsilon}(t)\right)-\widehat{x}^{\epsilon}(t)\right|^{2}=0 \\
\lim _{\rho \rightarrow 0} \sup _{0 \leq t \leq T} E\left|\rho^{-1}\left(p_{\rho}^{\epsilon}(t)-p^{\epsilon}(t)\right)-\widehat{p}^{\epsilon}(t)\right|^{2}=0 \\
\lim _{\rho \rightarrow 0} E\left[\int_{0}^{T} \int_{E} \mid \rho^{-1}\left(q_{\rho}^{\epsilon}(t, e)-q^{\epsilon}(t, e)\right)\right. \\
\left.-\left.\widehat{q}^{\epsilon}(t, e)\right|^{2} \lambda(d e) d t\right]=0 \\
\lim _{\rho \rightarrow 0} \sup _{0 \leq t \leq T} E\left|\rho^{-1}\left(y_{\rho}^{\epsilon}(t)-y^{\epsilon}(t)\right)-\widehat{y}^{\epsilon}(t)\right|^{2}=0 \\
\lim _{\rho \rightarrow 0} E\left[\int_{0}^{T}\left|\rho^{-1}\left(z_{\rho}^{\epsilon}(t)-z^{\epsilon}(t)\right)-\widehat{z}^{\epsilon}(t)\right|^{2} d t\right]=0 \\
\lim _{\rho \rightarrow 0} E\left[\int_{0}^{T} \int_{E} \mid \rho^{-1}\left(k_{\rho}^{\epsilon}(t, e)-k^{\epsilon}(t, e)\right)\right. \\
\left.-\left.\widehat{k}^{\epsilon}(t, e)\right|^{2} \lambda(d e) d t\right]=0
\end{gathered}
$$

It follows that

$$
\begin{gathered}
x_{\rho}^{\epsilon}(t)-x^{\epsilon}(t)=\rho \widehat{x}^{\epsilon}(t)+o(\rho), \\
p_{\rho}^{\epsilon}(t)-p^{\epsilon}(t)=\rho \widehat{p}^{\epsilon}(t)+o(\rho), \\
q_{\rho}^{\epsilon}(t, \cdot)-q^{\epsilon}(t, \cdot)=\rho \widehat{q}^{\epsilon}(t, \cdot)+o(\rho), \\
y_{\rho}^{\epsilon}(t)-y^{\epsilon}(t)=\rho \widehat{y}^{\epsilon}(t)+o(\rho), \\
z_{\rho}^{\epsilon}(t)-z^{\epsilon}(t)=\rho \widehat{z}^{\epsilon}(t)+o(\rho), \\
k_{\rho}^{\epsilon}(t, \cdot)-k^{\epsilon}(t, \cdot)=\rho \widehat{k}^{\epsilon}(t, \cdot)+o(\rho) .
\end{gathered}
$$

Furthermore, we get the following expansions:

$$
\begin{aligned}
&\left|x_{\rho}^{\epsilon}(0)-a\right|^{2}-\left|x^{\epsilon}(0)-a\right|^{2} \\
&= 2 \rho\left\langle x^{\epsilon}(0)-a, \widehat{x}^{\epsilon}(0)\right\rangle+o(\rho) \\
&\left|\psi\left(\xi_{p}^{\epsilon}\right)-\psi\left(\xi^{*}\right)+\epsilon\right|^{2}-\left|\psi\left(\xi^{\epsilon}\right)-\psi\left(\xi^{*}\right)+\epsilon\right|^{2} \\
&= 2 \rho\left[\psi\left(\xi^{\epsilon}\right)-\psi\left(\xi^{*}\right)+\epsilon\right]\left\langle\psi_{x}\left(\xi^{\epsilon}\right), \xi-\xi^{\epsilon}\right\rangle+o(\rho) \\
&\left|\gamma\left(y_{\rho}^{\epsilon}(0)\right)-\gamma\left(y^{*}(0)\right)+\epsilon\right|^{2}-\left|\gamma\left(y^{\epsilon}(0)\right)-\gamma\left(y^{*}(0)\right)+\epsilon\right|^{2} \\
&=2 \rho\left[\gamma\left(y^{\epsilon}(0)\right)-\gamma\left(y^{*}(0)\right)+\epsilon\right] \\
& \quad \times\left\langle\gamma_{y}\left(y^{\epsilon}(0)\right), \widehat{y}^{\epsilon}(0)\right\rangle+o(\rho)
\end{aligned}
$$

$$
\begin{aligned}
& \left|\int_{0}^{T} l_{\rho}^{\epsilon}(t, \cdot) d t-\int_{0}^{T} l^{*}(t, \cdot) d t+\epsilon\right|^{2} \\
& -\left|\int_{0}^{T} l^{\epsilon}(t, \cdot) d t-\int_{0}^{T} l^{*}(t, \cdot) d t+\epsilon\right|^{2} \\
& =2 \rho\left[\int_{0}^{T} l^{\epsilon}(t, \cdot) d t-\int_{0}^{T} l^{*}(t, \cdot) d t+\epsilon\right] \\
& \times\left[\int _ { 0 } ^ { T } \left(\left\langle l_{x}^{\epsilon}(t, \cdot), \widehat{x}^{\epsilon}(t)\right\rangle+\left\langle l_{y}^{\epsilon}(t, \cdot), \widehat{y}^{\epsilon}(t)\right\rangle\right.\right. \\
& +\left\langle l_{z}^{\epsilon}(t, \cdot), \widehat{z}^{\epsilon}(t)\right\rangle+\left\langle l_{k}^{\epsilon}(t, \cdot), \widehat{k}^{\epsilon}(t, \cdot)\right\rangle \\
& +\left\langle l_{p}^{\epsilon}(t, \cdot), \widehat{p}^{\epsilon}(t)\right\rangle \\
& \left.\left.+\left\langle l_{q}^{\epsilon}(t, \cdot), \widehat{q}^{\epsilon}(t, \cdot)\right\rangle\right) d t\right]+o(\rho),
\end{aligned}
$$

where

$$
\begin{aligned}
& l_{\rho}^{\epsilon}(t, \cdot):=l\left(t, x_{\rho}^{\epsilon}(t), y_{\rho}^{\epsilon}(t), z_{\rho}^{\epsilon}(t), k_{\rho}^{\epsilon}(t, \cdot), p_{\rho}^{\epsilon}(t), q_{\rho}^{\epsilon}(t, \cdot)\right), \\
& l^{\epsilon}(t, \cdot):=l\left(t, x^{\epsilon}(t), y^{\epsilon}(t), z^{\epsilon}(t), k^{\epsilon}(t, \cdot), p^{\epsilon}(t), q^{\epsilon}(t, \cdot)\right) .
\end{aligned}
$$

Given $\epsilon>0$, we discuss the following cases.

Case 1. There exists $\rho_{0}>0$ such that, for all $\rho \in\left(0, \rho_{0}\right)$,

$$
\begin{gathered}
\psi\left(\xi_{\rho}^{\epsilon}\right)-\psi\left(\xi^{*}\right)+\epsilon \geq 0, \\
\gamma\left(y_{\rho}^{\epsilon}(0)\right)-\gamma\left(y^{*}(0)\right)+\epsilon \geq 0, \\
\int_{0}^{T} l_{\rho}^{\epsilon}(t, \cdot) d t-\int_{0}^{T} l^{*}(t, \cdot) d t+\epsilon \geq 0 .
\end{gathered}
$$

In this case,

$$
\begin{aligned}
\lim _{\rho \rightarrow 0} & \frac{F_{\epsilon}\left(\xi_{\rho}^{\epsilon}\right)-F_{\epsilon}\left(\xi^{\epsilon}\right)}{\rho} \\
= & \lim _{\rho \rightarrow 0} \frac{1}{F_{\epsilon}\left(\xi_{\rho}^{\epsilon}\right)+F_{\epsilon}\left(\xi^{\epsilon}\right)} \frac{F_{\epsilon}^{2}\left(\xi_{\rho}^{\epsilon}\right)-F_{\epsilon}^{2}\left(\xi^{\epsilon}\right)}{\rho} \\
= & \frac{1}{F_{\epsilon}\left(\xi^{\epsilon}\right)}\left\{\left\langle x^{\epsilon}(0)-a, \widehat{x}^{\epsilon}(0)\right\rangle\right. \\
& +\left\langle\psi_{x}\left(\xi^{\epsilon}\right), \xi-\xi^{\epsilon}\right\rangle\left[\psi\left(\xi^{\epsilon}\right)-\psi\left(\xi^{*}\right)+\epsilon\right] \\
& +\left\langle\gamma_{y}\left(y^{\epsilon}(0)\right), \widehat{y}^{\epsilon}(0)\right\rangle\left[\gamma\left(y^{\epsilon}(0)\right)-\gamma\left(y^{*}(0)\right)+\epsilon\right]
\end{aligned}
$$




$$
\begin{aligned}
& +\left[\int _ { 0 } ^ { T } \left(\left\langle l_{x}^{\epsilon}(t, \cdot), \widehat{x}^{\epsilon}(t)\right\rangle+\left\langle l_{y}^{\epsilon}(t, \cdot), \widehat{y}^{\epsilon}(t)\right\rangle\right.\right. \\
& +\left\langle l_{z}^{\epsilon}(t, \cdot), \widehat{z}^{\epsilon}(t)\right\rangle+\left\langle l_{k}^{\epsilon}(t, \cdot), \widehat{k}^{\epsilon}(t, \cdot)\right\rangle \\
& \left.\left.\quad+\left\langle l_{p}^{\epsilon}(t, \cdot), \widehat{p}^{\epsilon}(t)\right\rangle+\left\langle l_{q}^{\epsilon}(t, \cdot), \widehat{q}^{\epsilon}(t, \cdot)\right\rangle\right) d t\right] \\
& \left.\times\left[\int_{0}^{T} l^{\epsilon}(t, \cdot) d t-\int_{0}^{T} l^{*}(t, \cdot) d t+\epsilon\right]\right\} .
\end{aligned}
$$

Setting

$$
\begin{gathered}
h_{0}^{\epsilon}=\frac{1}{F_{\epsilon}\left(\xi^{\epsilon}\right)}\left[x^{\epsilon}(0)-a\right], \\
h_{1}^{\epsilon}=\frac{1}{F_{\epsilon}\left(\xi^{\epsilon}\right)}\left[\psi\left(\xi^{\epsilon}\right)-\psi\left(\xi^{*}\right)+\epsilon\right] \geq 0, \\
h_{2}^{\epsilon}=\frac{1}{F_{\epsilon}\left(\xi^{\epsilon}\right)}\left[\gamma\left(y^{\epsilon}(0)\right)-\gamma\left(y^{*}(0)\right)+\epsilon\right] \geq 0, \\
h_{3}^{\epsilon}=\frac{1}{F_{\epsilon}\left(\xi^{\epsilon}\right)}\left[\int_{0}^{T} l^{\epsilon}(t, \cdot) d t-\int_{0}^{T} l^{*}(t, \cdot) d t+\epsilon\right] \geq 0
\end{gathered}
$$

and combining with (25), we get

$$
\begin{aligned}
& \left\langle h_{0}^{\epsilon}, \widehat{x}^{\epsilon}(0)\right\rangle+h_{1}^{\epsilon}\left\langle\psi_{x}\left(\xi^{\epsilon}\right), \xi-\xi^{\epsilon}\right\rangle \\
& +h_{2}^{\epsilon}\left\langle\gamma_{y}\left(y^{\epsilon}(0)\right), \widehat{y}^{\epsilon}(0)\right\rangle \\
& +h_{3}^{\epsilon} \int_{0}^{T}\left(\left\langle l_{x}^{\epsilon}(t, \cdot), \widehat{x}^{\epsilon}(t)\right\rangle\right. \\
& +\left\langle l_{y}^{\epsilon}(t, \cdot), \widehat{y}^{\epsilon}(t)\right\rangle+\left\langle l_{z}^{\epsilon}(t, \cdot), \widehat{z}^{\epsilon}(t)\right\rangle \\
& +\left\langle l_{k}^{\epsilon}(t, \cdot), \widehat{k}^{\epsilon}(t, \cdot)\right\rangle+\left\langle l_{p}^{\epsilon}(t, \cdot), \widehat{p}^{\epsilon}(t)\right\rangle \\
& \left.+\left\langle l_{q}^{\epsilon}(t, \cdot), \widehat{q}^{\epsilon}(t, \cdot)\right\rangle\right) d t \\
& \geq-\sqrt{\sqrt{3} \epsilon}\left[E\left|\xi-\xi^{\epsilon}\right|^{2}\right]^{1 / 2} \cdot
\end{aligned}
$$

Case 2. There exists a positive sequence $\left\{\rho_{n}\right\}$ satisfying $\rho_{n} \rightarrow$ 0 , such that

$$
\begin{gathered}
\psi\left(\xi_{\rho_{n}}^{\epsilon}\right)-\psi\left(\xi^{*}\right)+\epsilon \leq 0, \\
\gamma\left(y_{\rho_{n}}^{\epsilon}(0)\right)-\gamma\left(y^{*}(0)\right)+\epsilon \leq 0, \\
\int_{0}^{T} l_{\rho}^{\epsilon}(t, \cdot) d t-\int_{0}^{T} l^{*}(t, \cdot) d t+\epsilon \leq 0 .
\end{gathered}
$$

In this case, from the definition of $F_{\epsilon}, F_{\epsilon}\left(\xi_{\rho_{n}}^{\epsilon}\right)=\left\{\mid x_{\rho_{n}}(0)-\right.$ $\left.\left.a\right|^{2}\right\}^{1 / 2}$. Due to $F_{\epsilon}(\cdot)$ being continuous, we have $F_{\epsilon}\left(\xi^{\epsilon}\right)=$ $\left\{\left|x^{\epsilon}(0)-a\right|^{2}\right\}^{1 / 2}$.
Thus,

$$
\begin{aligned}
\lim _{n \rightarrow \infty} & \frac{F_{\epsilon}\left(\xi_{\rho_{n}}^{\epsilon}\right)-F_{\epsilon}\left(\xi^{\epsilon}\right)}{\rho_{n}} \\
& =\lim _{n \rightarrow \infty} \frac{1}{F_{\epsilon}\left(\xi_{\rho_{n}}^{\epsilon}\right)+F_{\epsilon}\left(\xi^{\epsilon}\right)} \frac{F_{\epsilon}^{2}\left(\xi_{\rho_{n}}^{\epsilon}\right)-F_{\epsilon}^{2}\left(\xi^{\epsilon}\right)}{\rho_{n}} \\
& =\frac{\left\langle x^{\epsilon}(0)-a, \widehat{x}^{\epsilon}(0)\right\rangle}{F_{\epsilon}\left(\xi^{\epsilon}\right)} .
\end{aligned}
$$

Similarly, from (25),

$$
\left\langle h_{0}^{\epsilon}, \widehat{x}^{\epsilon}(0)\right\rangle \geq-\sqrt{\sqrt{3} \epsilon}\left[E\left|\xi-\xi^{\epsilon}\right|^{2}\right]^{1 / 2},
$$

where $h_{0}^{\epsilon}=\left(1 / F_{\epsilon}\left(\xi^{\epsilon}\right)\right)\left[x^{\epsilon}(0)-a\right], h_{1}^{\epsilon}=0, h_{2}^{\epsilon}=0, h_{3}^{\epsilon}=0$.

We point out that, for the other six cases, the similar (33) also holds.

So, in any way, we always have that (i) (33) holds, (ii) $h_{1}^{\epsilon} \geq$ $0, h_{2}^{\epsilon} \geq 0, h_{3}^{\epsilon} \geq 0$, and (iii) $\left|h_{0}^{\epsilon}\right|^{2}+\left|h_{1}^{\epsilon}\right|^{2}+\left|h_{2}^{\epsilon}\right|^{2}+\left|h_{3}^{\epsilon}\right|^{2}=1$.

Therefore, we obtain the existence of a convergent subsequence of $\left(h_{0}^{\epsilon}, h_{1}^{\epsilon}, h_{2}^{\epsilon}, h_{3}^{\epsilon}\right)$ whose limit is denoted by $\left(h_{0}, h_{1}, h_{2}, h_{3}\right)$. Because of $d\left(\xi^{\epsilon}, \xi^{*}\right)<\epsilon$, we know $\xi^{\epsilon} \rightarrow \xi^{*}$ in $U$, as $\epsilon \rightarrow 0$. According to the regularity of the solutions of BSDEs with jumps, as $\epsilon \rightarrow 0$, we have

$$
\begin{aligned}
& \left(\widehat{x}^{\epsilon}(\cdot), \widehat{p}^{\epsilon}(\cdot), \widehat{q}^{\epsilon}(\cdot, \cdot)\right) \longrightarrow(\widehat{x}(\cdot), \widehat{p}(\cdot), \widehat{q}(\cdot, \cdot)), \\
& \left(\widehat{y}^{\epsilon}(t), \widehat{z}^{\epsilon}(\cdot), \widehat{k}^{\epsilon}(\cdot, \cdot)\right) \longrightarrow(\widehat{y}(\cdot), \widehat{z}(\cdot), \widehat{k}(\cdot, \cdot)) .
\end{aligned}
$$

By letting $\epsilon \rightarrow 0$ in (33), we deduce the variational inequality (23).

2.5. Maximum Principle. In this subsection, we will prove the stochastic maximum principle. First, the adjoint equation is introduced as follows:

$$
\begin{aligned}
& d m(t)= {\left[b_{x}^{*}(t, \cdot)^{T} m(t)+g_{x}^{*}(t, \cdot)^{T} n(t)+h_{3} l_{x}^{*}(t, \cdot)\right] d t } \\
&+\left[b_{p}^{*}(t, \cdot)^{T} m(t)+g_{p}^{*}(t, \cdot)^{T} n(t)+h_{3} l_{p}^{*}(t, \cdot)\right] d B_{t} \\
&+\int_{E}\left[b_{q}^{*}(t, e)^{T} m(t)+g_{q}^{*}(t, e)^{T} n(t)\right. \\
&\left.+h_{3} l_{q}^{*}(t, e)\right] \tilde{\mu}(d t d e), \\
& m(0)=h_{0}, \\
& d n(t)=\left[b_{y}^{*}(t, \cdot)^{T} m(t)+g_{y}^{*}(t, \cdot)^{T} n(t)+h_{3} l_{y}^{*}(t, \cdot)\right] d t \\
&+\left[b_{z}^{*}(t, \cdot)^{T} m(t)+g_{z}^{*}(t, \cdot)^{T} n(t)+h_{3} l_{z}^{*}(t, \cdot)\right] d B_{t} \\
&+\int_{E}\left[b_{k}^{*}(t, \cdot)^{T} m(t)+g_{k}^{*}(t, e)^{T} n(t)\right. \\
&\left.+h_{3} l_{k}^{*}(t, \cdot)\right] \tilde{\mu}(d t d e), \\
& \\
& \quad n(0)=h_{2} \gamma_{y}\left(y^{*}(0)\right), \quad t \in[0, T] .
\end{aligned}
$$


We refer to the solution processes $(m(\cdot), n(\cdot))$ of $(38)$ as the adjoint processes. In fact, there are two coupled SDEs with jumps in (38). The adjoint equation (38) can be easily solved when we regard $(m(\cdot), n(\cdot))$ as a whole.

The following is the main result of this paper.

Theorem 7. Assume $\left(\mathrm{H}_{1}\right)-\left(\mathrm{H}_{4}\right)$. Let $\xi^{*}$ be optimal to Problem II and let $\left(x^{*}(\cdot), p^{*}(\cdot), q^{*}(\cdot, \cdot), y^{*}(\cdot), z^{*}(\cdot), k^{*}(\cdot, \cdot)\right)$ be the corresponding state of (13). Then, there exist $h_{0} \in \mathbb{R}^{n}, h_{1}, h_{2}, h_{3} \geq 0$, and $\left|h_{0}\right|+\left|h_{1}\right|+\left|h_{2}\right|+\left|h_{3}\right| \neq 0$ such that, for any $\xi \in U$,

$$
\left\langle m(T)+\Phi_{x}\left(\xi^{*}\right)^{T} n(T)+h_{1} \psi_{x}\left(\xi^{*}\right), \xi-\xi^{*}\right\rangle \geq 0, \quad \text { a.s., }
$$

where $(m(\cdot), n(\cdot))$ is the solution of $(38)$.

Proof. Applying Itô's formula to $\langle m(t), \widehat{x}(t)\rangle+\langle n(t), \widehat{y}(t)\rangle$, we get

$$
\begin{aligned}
d\langle m(t), \widehat{x}(t)\rangle+\langle n(t), \widehat{y}(t)\rangle & \\
=[ & h_{3} l_{x}^{*}(t, \cdot)^{T} \widehat{x}(t)+h_{3} l_{p}^{*}(t, \cdot)^{T} \widehat{p}(t) \\
& +h_{3} \int_{E} l_{q}^{*}(t, e)^{T} \widehat{q}(t, e) \lambda(d e) \\
& +h_{3} l_{y}^{*}(t, \cdot)^{T} \widehat{y}(t)+h_{3} l_{z}^{*}(t, \cdot)^{T} \widehat{z}(t) \\
& \left.+h_{3} \int_{E} l_{k}^{*}(t, e)^{T} \widehat{k}(t, e) \lambda(d e)\right] d t+\{\cdots\} d B_{t} .
\end{aligned}
$$

Integrating from 0 to $T$, taking expectation, we obtain

$$
\begin{aligned}
E[\langle m(T) & \left.\left.+\Phi_{x}\left(\xi^{*}\right)^{T} n(T), \xi-\xi^{*}\right\rangle\right] \\
= & \left\langle h_{0}, \widehat{x}(0)\right\rangle+h_{2}\left\langle\gamma_{y}\left(y^{*}(0)\right), \widehat{y}(0)\right\rangle \\
& +h_{3} \int_{0}^{T}\left\langle l_{x}^{*}(t, \cdot), \widehat{x}(t)\right\rangle d t \\
& +h_{3} \int_{0}^{T}\left\langle l_{p}^{*}(t, \cdot), \widehat{p}(t)\right\rangle d t \\
& +h_{3} \int_{0}^{T}\left\langle l_{q}^{*}(t, \cdot), \widehat{q}(t, e)\right\rangle \lambda(d e) d t \\
& +h_{3} \int_{0}^{T}\left\langle l_{y}^{*}(t, \cdot), \widehat{y}(t)\right\rangle d t \\
& +h_{3} \int_{0}^{T}\left\langle l_{z}^{*}(t, \cdot), \widehat{z}(t)\right\rangle d t \\
& +h_{3} \int_{0}^{T}\left\langle l_{k}^{*}(t, \cdot), \widehat{k}(t, e)\right\rangle \lambda(d e) d t .
\end{aligned}
$$

Combined with the variational inequality (23), we have

$$
E\left[\left\langle m(T)+\Phi_{x}\left(\xi^{*}\right)^{T} n(T)+h_{1} \psi_{x}\left(\xi^{*}\right), \xi-\xi^{*}\right\rangle\right] \geq 0
$$

Since $\xi \in U$ is arbitrary, we know, for any $\xi \in K$, that

$$
\left\langle m(T)+\Phi_{x}\left(\xi^{*}\right)^{T} n(T)+h_{1} \psi_{x}\left(\xi^{*}\right), \xi-\xi^{*}\right\rangle \geq 0, \quad \text { a.s. }
$$

Denote $\partial U$ by the boundary of $U$. Define $\Omega_{0}:=\{\omega \in \Omega \mid$ $\left.\xi^{*}(\omega) \in \partial U\right\}$.

From Theorem 7, we have the following.

Corollary 8. Under the assumptions of Theorem 7, for each $\xi \in U$, one has

$$
\begin{array}{r}
\left\langle m(t)+\Phi_{x}\left(\xi^{*}\right)^{T} n(t)+h_{1} \psi_{x}\left(\xi^{*}\right), \xi-\xi^{*}\right\rangle \geq 0, \\
\text { a.s. on } \Omega_{0}, \\
m(t)+\Phi_{x}\left(\xi^{*}\right)^{T} n(t)+h_{1} \psi_{x}\left(\xi^{*}\right)=0, \\
\text { a.s. on } \Omega_{0}^{c} .
\end{array}
$$

\section{Application: Stochastic LQ Control with Terminal State Constraints}

Now we consider a linear quadratic (LQ, for short) control problem of FBSDEs with jumps. For convenience, we assume $m=n=d=1$.

Consider the following linear control system:

$$
\begin{gathered}
d x(t)=(x(t)-3 y(t)+k(t, \cdot)+u(t)+2 v(t, \cdot)) d t \\
+(3 x(t)+3 z(t)+2 k(t, \cdot)+2 u(t)) d B_{t} \\
+\int_{E}(x(t)-y(t)-2 z(t)-2 k(t, e) \\
+v(t, e)) \tilde{\mu}(d t d e), \\
x(0)=a, \\
d y(t)=-(x(t)+y(t)+3 z(t)+k(t, \cdot) \\
+3 u(t)+2 v(t, \cdot)) d t+z(t) d B_{t} \\
+\int_{E} k(t, e) \tilde{\mu}(d t d e), \\
y(T)=x(T), \quad t \in[0, T] .
\end{gathered}
$$

The objective of our control problem is to minimize the following cost functional:

$$
J(u(\cdot), v(\cdot, \cdot))=E\left[x(T)^{2}+y(0)^{2}\right]
$$

subject to $u(\cdot) \in M^{2}(0, T ; \mathbb{R}), v(\cdot, \cdot) \in K_{\lambda}^{2}(0, T ; \mathbb{R}), x(T) \in \mathbb{R}^{+}$, a.s. 
Following the procedures in previous section, let $3 x(t)+$ $3 z(t)+2 k(t, \cdot)+2 u(t)=p(t), x(t)-y(t)-2 z(t)-2 k(t, e)+$ $v(t, e)=q(t, e)$, and treat $p(\cdot), q(\cdot, \cdot)$ as the controls; then, the backward control system can be rewritten as

$$
\begin{aligned}
& d x(t)=(-\frac{5}{2} x(t)-y(t)+\frac{5}{2} z(t)+4 k(t, \cdot) \\
&\left.+\frac{p(t)}{2}+2 q(t, \cdot)\right) d t+p(t) d B_{t} \\
&+\int_{E} q(t, e) \tilde{\mu}(d t d e), \\
& d y(t)=-\left(-\frac{11}{2} x(t)+3 y(t)+7 z(t)+2 k(t, \cdot)\right. \\
&\left.+\frac{3}{2} p(t)-\frac{5}{2} q(t, \cdot)\right) d t+z(t) d B_{t} \\
&+ \int_{E} k(t, e) \tilde{\mu}(d t d e), \\
& y(T)=\xi .
\end{aligned}
$$

Accordingly, the equivalent control problem is

$$
\begin{aligned}
& \text { Minimize } \quad J(u(\cdot), v(\cdot, \cdot))=E\left[\xi^{2}+y(0)^{2}\right], \\
& \text { subject to } \quad \xi \in \mathbb{R}^{+}, \quad x_{0}^{\xi}=a .
\end{aligned}
$$

Then, from Theorem 7, if $\xi^{*}$ is optimal, there exist $h_{1}, h_{0} \in \mathbb{R}$, with $h_{0} \geq 0$ and $\left|h_{0}\right|+\left|h_{1}\right| \neq 0$ such that, for any $\xi \geq 0$,

$$
\left(m(T)+n(T)+2 h_{1} \xi^{*}\right)\left(\xi-\xi^{*}\right) \geq 0, \quad \text { a.s. },
$$

where $(m(\cdot), n(\cdot))$ is the solution of the following equation:

$$
\begin{aligned}
& d m(t)=\left(\frac{5}{2} m(t)-\frac{11}{2} n(t)\right) d t \\
&+\left(-\frac{1}{2} m(t)+\frac{3}{2} n(t)\right) d B_{t} \\
&+\int_{E}\left(-2 m(t)-\frac{5}{2} n(t)\right) \tilde{\mu}(d t d e), \\
& m(0)=h_{0}, \\
& d n(t)=(m(t)+3 n(t)) d t+\left(-\frac{5}{2}+7 n(t)\right) d B_{t} \\
&+ \int_{E}(-4 m(t)+2 n(t)) \tilde{\mu}(d t d e), \\
& n(0)=2 h_{2} y^{*}(0) .
\end{aligned}
$$

Denote $\Omega_{0}:=\left\{\omega \in \Omega \mid \xi^{*}(\omega)=0\right\}$. From the arbitrariness of $\xi$, we get the following necessary condition of the optimal control $\xi^{*}$ :

$$
\begin{aligned}
& m(T)+n(T)+2 h_{1} \xi^{*} \geq 0, \quad \text { a.s. on } \Omega_{0}, \\
& m(T)+n(T)+2 h_{1} \xi^{*}=0, \quad \text { a.s. on } \Omega_{0}^{c},
\end{aligned}
$$

where $(m(\cdot), n(\cdot))$ is the solution of $(50)$.

\section{Conflict of Interests}

The author declares that there is no conflict of interests regarding the publication of this paper.

\section{Acknowledgments}

This work was supported by National Natural Science Foundation of China (nos. 11171187, 11222110, and 11221061); supported by Shandong Province (no. JQ201202); supported by Program for New Century Excellent Talents in University of China; and supported by Postdoctoral Scientific Research Project of Jilin Province (no. RB201357).

\section{References}

[1] É. Pardoux and S. G. Peng, "Adapted solution of a backward stochastic differential equation," Systems \& Control Letters, vol. 14, no. 1-2, pp. 55-61, 1990.

[2] H. Kushner, "Necessary conditions for continuous parameter stochastic optimization problems," SIAM Journal on Control and Optimization, vol. 10, pp. 550-565, 1972.

[3] J.-M. Bismut, "An introductory approach to duality in optimal stochastic control," SIAM Journal on Control and Optimization, vol. 20, no. 1, pp. 62-78, 1978.

[4] A. Bensoussan, "Lectures on stochastic control," in Nonlinear Filtering and Stochastic Control, Lecture Notes in Mathematics, part I, pp. 1-39, Springer, New York, NY, USA, 1983.

[5] U. Haussmann, A Stochastic Maximum Principle for Optimal Control of Diffusions, vol. 151 of Pitman Research Notes in Mathematics, Wiley John \& Sons, New York, NY, USA, 1986.

[6] Y. Hu, "Maximum principle of optimal control for Markov processes," Acta Mathematica Sinica, vol. 33, pp. 43-56, 1990.

[7] S. G. Peng, "A general stochastic maximum principle for optimal control problems," SIAM Journal on Control and Optimization, vol. 28, no. 4, pp. 966-979, 1990.

[8] S. G. Peng, "Backward stochastic differential equations and applications to optimal control," Applied Mathematics and Optimization, vol. 27, no. 4, pp. 125-144, 1993.

[9] B. Øksendal and A. Sulem, "Maximum principles for optimal control of forward-backward stochastic differential equations with jumps," SIAM Journal on Control and Optimization, vol. 48, no. 5, pp. 2945-2976, 2009.

[10] J. M. Yong and X. Y. Zhou, Stochastic Controls: Hamiltonian Systems and HJB Equations, vol. 43 of Applications of Mathematics, Springer, New York, NY, USA, 1999.

[11] W. S. Xu, "Stochastic maximum principle for optimal control problem of forward and backward system," Journal of the Australian Mathematical Society B, vol. 37, no. 2, pp. 172-185, 1995.

[12] N. El Karoui, S. Peng, and M. C. Quenez, "A dynamic maximum principle for the optimization of recursive utilities under constraints," The Annals of Applied Probability, vol. 11, no. 3, pp. 664-693, 2001.

[13] S. L. Ji and S. G. Peng, "Terminal perturbation method for the backward approach to continuous time mean-variance portfolio selection," Stochastic Processes and their Applications, vol. 118, no. 6, pp. 952-967, 2008. 
[14] S. L. Ji and X. Y. Zhou, "A maximum principle for stochastic optimal control with terminal state constraints, and its applications," Communications in Information and Systems, vol. 6, no. 4, pp. 321-337, 2006.

[15] S. L. Ji and Z. Wu, "The maximum principle for one kind of stochastic optimization problem and application in dynamic measure of risk," Acta Mathematica Sinica, vol. 23, no. 12, pp. 2189-2204, 2007.

[16] S. L. Ji and X. Y. Zhou, "The Neyman-Pearson lemma under g-probability," Comptes Rendus de l'Académie des Sciences, vol. 346, no. 3-4, pp. 209-212, 2008.

[17] S. L. Ji and X. Y. Zhou, "A generalized Neyman-Pearson lemma under g-probabilities," Probability Theory and Related Fields, vol. 148, no. 3-4, pp. 645-669, 2010.

[18] S. L. Ji, "Dual method for continuous-time Markowitz's problems with nonlinear wealth equations," Journal of Mathematical Analysis and Applications, vol. 366, no. 1, pp. 90-100, 2010.

[19] J. T. Shi, "Necessary conditions for optimal control of forwardbackward stochastic systems with random jumps," International Journal of Stochastic Analysis, vol. 2012, Article ID 258674, 50 pages, 2012.

[20] J. T. Shi and Z. Wu, "Necessary condition for optimal control of fully coupled forward-backward stochastic system with random jumps," in Proceedings of the 31th Chinese Control Conference, pp. 1620-1627, Hefei, China, 2012.

[21] R. Situ, "A maximum principle for optimal controls of stochastic with random jumps," in Proceedings of the National Conference on Control Theory and Its Applications, Qingdao, China, October 1991.

[22] S. J. Tang and X. J. Li, "Necessary conditions for optimal control of stochastic systems with random jumps," SIAM Journal on Control and Optimization, vol. 32, no. 5, pp. 1447-1475, 1999.

[23] J. T. Shi and Z. Wu, "Maximum principle for forward-backward stochastic control system with random jumps and applications to finance," Journal of Systems Science \& Complexity, vol. 23, no. 2, pp. 219-231, 2010.

[24] T. Bielecki, H. Jin, S. Pliska, and X. Y. Zhou, "Continuoustime mean-variance portfolio selection with bankruptcy prohibition," Mathematical Finance, vol. 15, no. 2, pp. 213-244, 2005.

[25] C. Bernard, S. L. Ji, and W. D. Tian, "An optimal insurance design problem under Knightian uncertainty," Decisions in Economics and Finance, vol. 36, no. 2, pp. 99-124, 2013.

[26] S. L. Ji and Q. M. Wei, "An overview on the principal-agent problems in continuous time," in Real Options, Ambiguity, Risk and Insurance, A. Bensoussan, S. Peng, and J. Sung, Eds., Studies in Probability, Optimization and Statistics 5, IOS Press, Amsterdam, The Netherlands, 2013.

[27] S. L. Ji and Q. M. Wei, "A maximum principle for fully coupled forward-backward stochastic control systems with terminal state constraints," Journal of Mathematical Analysis and Applications, vol. 407, no. 2, pp. 200-210, 2013.

[28] S. L. Ji, Q. M. Wei, and X. M. Zhang, "A maximum principle for controlled time-symmetric forward-backward doubly stochastic differential equation with initial-terminal sate constraints," Abstract and Applied Analysis, vol. 2012, Article ID 537376, 29 pages, 2012.

[29] L. Epstein and S. L. Ji, "Ambiguous volatility and asset pricing in continuous time," The Review of Financial Studies, vol. 26, no. 7, pp. 1740-1786, 2013.

[30] L. Epstein and S. L. Ji, "Ambiguous volatility, possibility and utility in continuous time," Journal of Mathematical Economics, 2013.
[31] Z. Wu, "Fully coupled FBSDE with Brownian motion and Poisson process in stopping time duration," Journal of the Australian Mathematical Society, vol. 74, no. 2, pp. 249-266, 2003.

[32] G. Barles, R. Buckdahn, and E. Pardoux, "Backward stochastic differential equations and integral-partial differential equations," Stochastics and Stochastics Reports, vol. 60, pp. 57-83, 1997.

[33] I. Ekeland, "On the variational principle," Journal of Mathematical Analysis and Applications, vol. 47, pp. 324-353, 1974. 


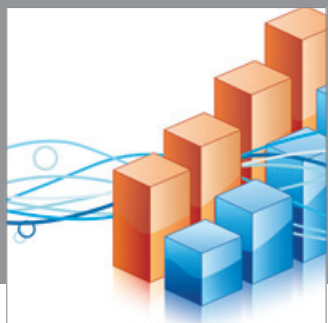

Advances in

Operations Research

mansans

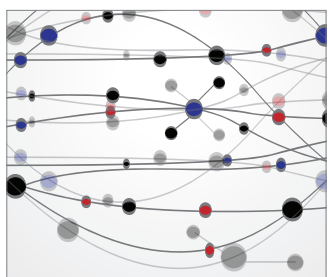

The Scientific World Journal
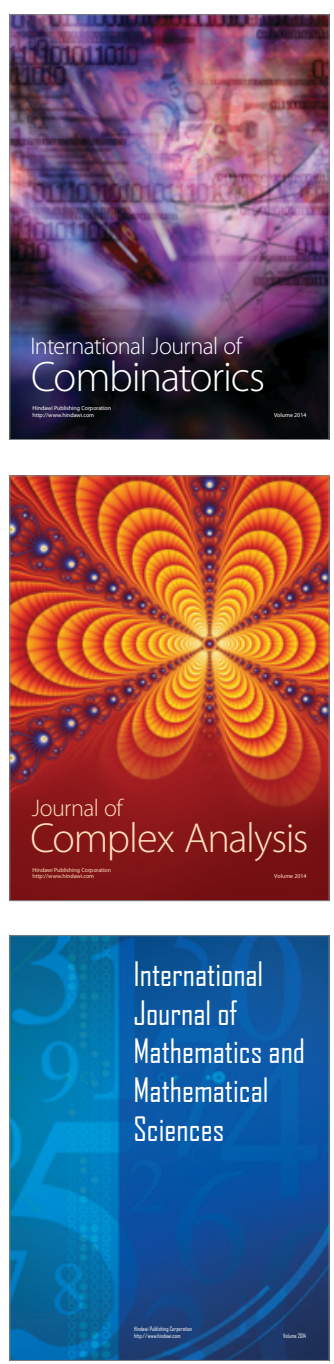
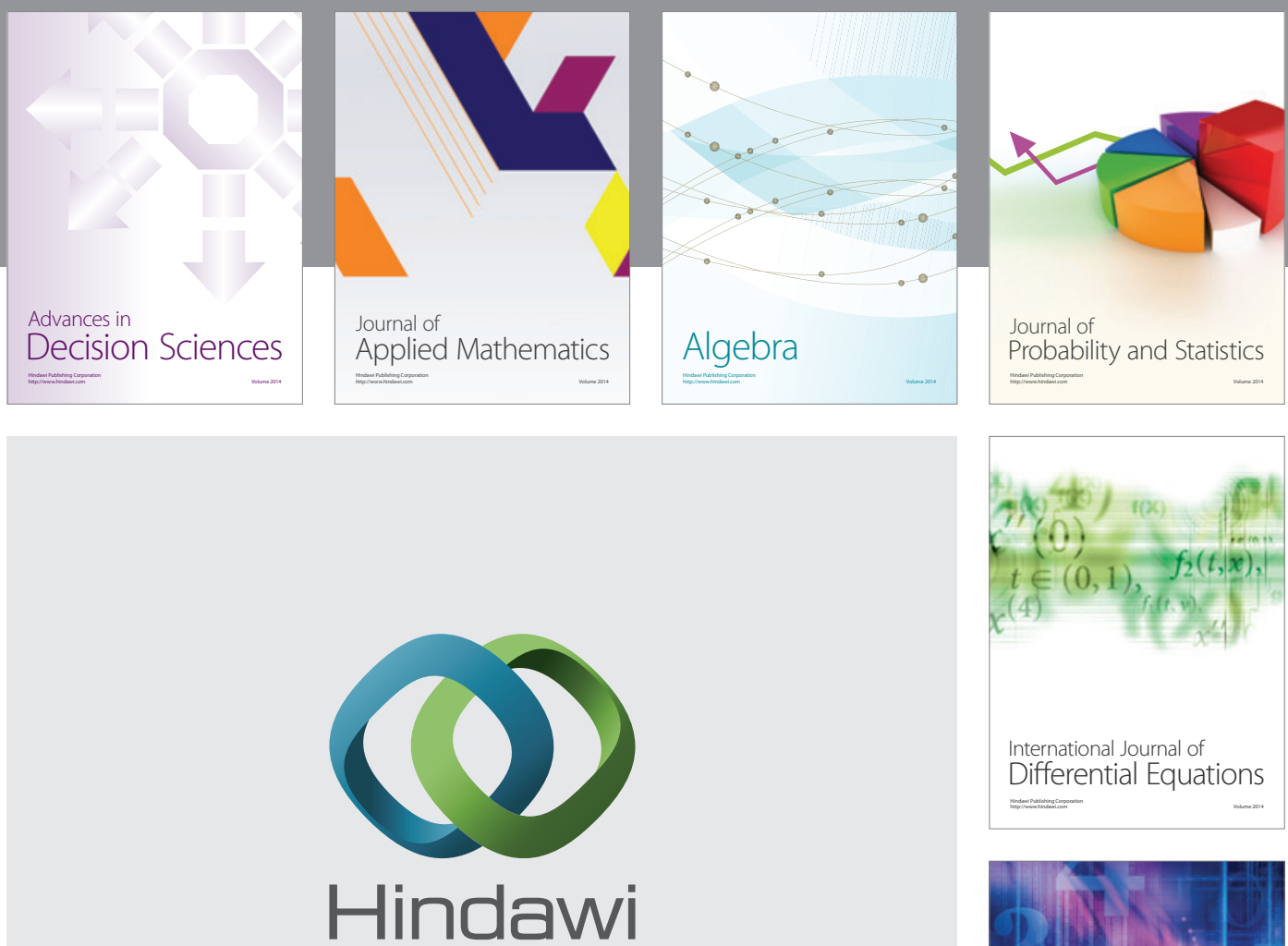

Submit your manuscripts at http://www.hindawi.com
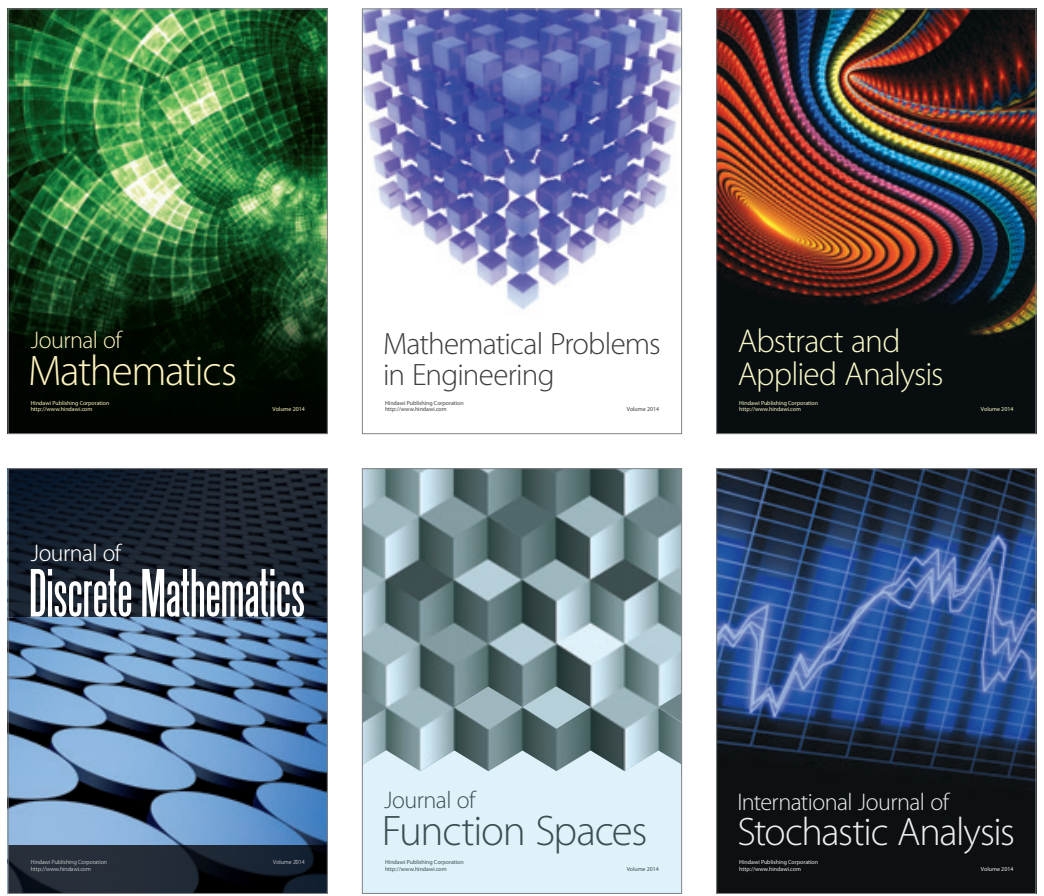

Journal of

Function Spaces

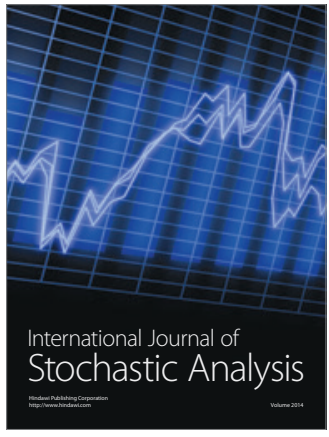

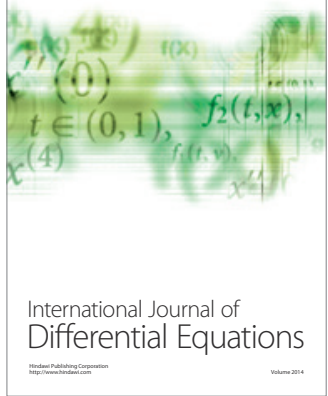
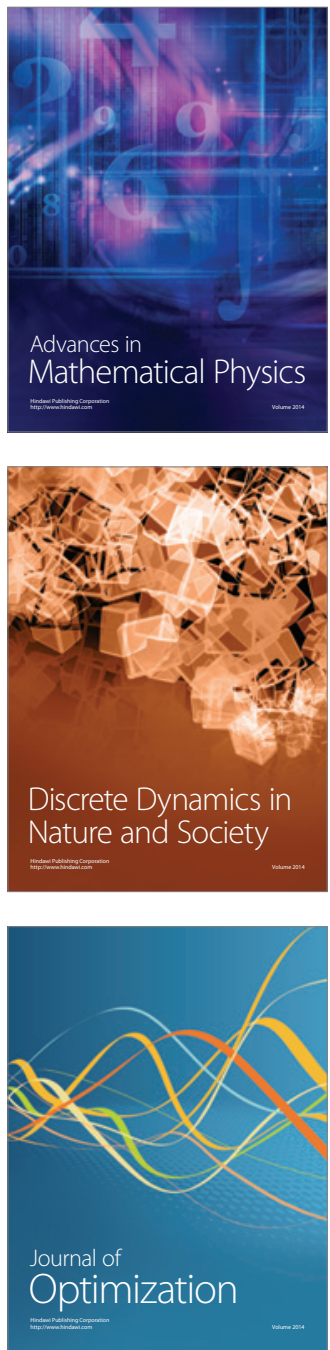